\title{
The impact of thyroid autoimmunity (TPOAb) on bone density and fracture risk in postmenopausal women
}

\author{
Snezana P. Polovina, ${ }^{1}$ Dragana Miljic, ${ }^{1,2}$ Sladjana Zivojinovic, ${ }^{3}$ Natasa Milic, ${ }^{4}$ \\ Dragan Micic, ${ }^{2}$ Vera Popovic Brkic ${ }^{2}$
}

${ }^{1}$ Clinic for Endocrinology, Diabetes and Diseases of Metabolism, Clinical Center of Serbia, Belgrade, Serbia; ${ }^{2}$ Faculty of Medicine, University of Belgrade, Serbia; ${ }^{3}$ Institute for Rheumatology, Belgrade, Serbia; ${ }^{4}$ Institute for Medical Statistic and Informatics, Medical Faculty, University of Belgrade, Serbia

\begin{abstract}
OBJECTIVE: Skeletal development, linear growth, cartilage biology and bone turnover are highly dependent on the activity of thyroid hormones. Thyroid dysfunction affects the skeleton, and autoimmune thyroid disease, manifesting as a chronic inflammatory condition, may be an important contributing factor to impaired bone quality in these patients. MATERIALS AND METHODS: Measurement of TSH, FT4, TPOAb and bone mineral density and FRAX score calculations were performed in 189 postmenopausal women (110 euthyroid women and 79 women diagnosed with subclinical hypothyroidism) divided into subgroups according to the presence of TPOAb. RESULTS: In multivariate logistic regression analysis only TPOAb were found to be significantly related to fracture, independently of TSH values $(p=0.018 ; 0 R=7.800$; 95\% CI 1.424-42.721). Lower bone mineral density and FRAX score for hip and for major osteoporotic fractures were associated with the presence of TPOAb in euthyroid postmenopausal women in an unadjusted logistic regression model, as well as in a model adjusted for age, BMI and smoking. TSH was a better predictive factor for fractures in women with subclinical hypothyroidism (FRAX main $p<0.001 ; 95 \%$ CI for SE $0.858-0.959$ and FRAX hip $p<0.001$; 95\% CI for SE 0.628-0.854). CONCLUSION: Autoimmune thyroid disease is associated with decreased bone mineral density at both spine and hip and risk of future fracture incidence in euthyroid postmenopausal women. Presence of TPOAb is a potential marker of higher fracture risk in these patients. However, in subclinical hypothyroidism, TSH is a better indicator of future fragility fractures than TPOAb.
\end{abstract}

Key words: Thyroid autoimmunity, AITD, TPOAb, Bone quality, Fracture risk, FRAX

\section{INTRODUCTION}

Skeletal development, linear growth, cartilage biology and bone turnover are highly dependent on

Address for correspondence:

Snezana P. Polovina, E-mail: snezanapolovina@gmail.com

Received: 05-12-2016, Accepted: 10-02-2017 thyroid function, with thyroid dysfunction seriously affecting the skeleton. It has been demonstrated that hyperthyroidism increases the risk of bone fractures due to thyroxine's impact on osteoclastic activity, while hypothyroidism affects bone quality through reduced bone remodeling. In addition to the effects of thyroid hormones on bones, TSH has an impact 
of its own on bone turnover. Indeed, TSH in the low-normal range correlates with a high incidence of vertebral fractures, independently of age, body mass index and levels of triiodothyronine and thyroxine. ${ }^{1}$ While a deficiency of thyroid hormones in adulthood is thought to increase bone mineral density despite increased bone quantity, hypothyroidism is accompanied by increased fracture risk. ${ }^{2}$ Meanwhile, patients with the subclinical form of thyroid dysfunction have poorer bone quality. In subclinical thyroid diseases, TSH outside the reference range is an independent predictive factor for vertebral fractures. ${ }^{3-5}$ In postmenopausal women, even the subclinical form of thyroid dysfunction can potentially increase the risk of fragility fractures. ${ }^{6}$ In addition, age and thyroid autoimmunity could predict a vertebral fracture in euthyroid postmenopausal women. ${ }^{7}$

Autoimmune thyroid disease (AITD), like many other chronic autoimmune inflammatory diseases, may be a contributing factor to poor bone quality. Investigations during the past ten years have provided strong evidence of the link between systemic inflammation and bone quality. ${ }^{7}$ Disturbance in osteclastic activity in new bone formation might be a consequence of chronic inflammatory autoimmune disorders leading to lower bone mineral density. Immune mediators found to play a role in most autoimmune disorders could also be involved in promoting bone loss in AITD. ${ }^{8}$

Although bone mineral density (BMD) measured by dual-energy-X-ray absorptiometry-(DXA) is the gold standard diagnostic tool for bone quality, the World Health Organization Collaborating Centre for Metabolic Bone Diseases in Sheffield (UK) proposed the FRAX score as a prognostic tool for assessing the risk of the low energy fractures. ${ }^{3}$ Other assessment tools, e.g. MORES, had greater sensitivity but lower specificity. ${ }^{9}$ The FRAX calculator is not available in all countries worldwide, thus the WHO has recommended using the FRAX model from a surrogate country with similarity in country-specific mortality rates. ${ }^{10}$ Identification of persons at high risk of low energy fractures, particularly among postmenopausal women, is of major importance to prevent fractures in the future. The aim of our study was to investigate the impact of thyroid autoimmunity on fracture risk in euthyroid postmenopausal women.

\section{MATERIAL AND METHODS}

A cross-sectional study with measurement of TSH, FT4, TPOAb and bone mineral density as well as FRAX score calculations (10-year estimated risk for bone fracture) were performed in 189 postmenopausal women (110 euthyroid women and 79 women diagnosed with subclinical hypothyroidism) divided into subgroups according to the presence of TPOAb. Age range was 41-74 years; menopause onset range was $35-57$ years.

Other clinical characteristics of the investigated subjects are presented in Table 1. The inclusion criteria for the study were: postmenopausal women 40-75 years of age with or without TPOAb and with TSH within the reference range or elevated but with fT4 in the euthyroid range. Exclusion criteria were: history of previous thyroid dysfunction, steroid therapy for more than 6 months, systemic inflammatory disease and inflammatory bowel disease, vitamin D levels less than $50 \mathrm{nmol} / \mathrm{L}$, high level of parathyroid hormone, alkaline phosphatase or serum calcium outside the reference range. All participants voluntarily filled in a questionnaire about parental hip fractures, previous fractures, current cigarette smoking and onset of menopause. For "previous fractures", we used the University of Sheffield FRAX score calculator definition: "fractures in adult life occurring spontaneously or a fracture arising from trauma which, in a healthy individual, would not have resulted in fracture". ${ }^{11}$ The investigation was performed after obtaining the approval of the local Ethics Committee.

TSH and fT4 were measured by the CMIA (chemiluminescent microparticle immunoassay) method by Abbott on ARCHITECT ci8200. The reference range for TSH was $0.35-4.94 \mathrm{mIU} / \mathrm{mL}$ with an analytical sensitivity of $\leq 0.1 \mu \mathrm{IU} / \mathrm{mL}$. TPOAb were measured by CMIA (chemiluminescent microparticle immunoassay) by Abbott for the quantitative determination of the IgG class of thyroid peroxidase autoantibodies in human serum and plasma on the ARCHITECT $i$ system. Normal values were $<5.61 \mathrm{IU} / \mathrm{mL}$. Cut-off for TPO “+" was $\geq 10 \mathrm{IU} / \mathrm{ml}$. 25-hydroxy vitamin D was measured by the ECLIA (electrochemiluminiscence immunoassay) method on Cobas e601. Cut-off for vitamin D insufficiency was $50 \mathrm{nmol} / \mathrm{L}(<50 \mathrm{nmol} / \mathrm{L}-$ deficiency, $>75 \mathrm{nmol} / \mathrm{L}$-normal level). Bone mineral 
density was measured by the Hologic dual energy $\mathrm{X}$-ray bone densitometer. Measuring was done on the left femoral neck and lumbar spine. BMD data are presented as standard deviation (SD) in T-score.

In order to assess risk of fragility fractures we used the FRAX score calculator as a reliable tool promoted by the National Osteoporosis Foundation. ${ }^{4}$ Fracture risk was calculated by the FRAX score assessment for Turkey in accordance with the WHO recommendation for use of a surrogate country calculation tool, based on country-specific mortality rates. ${ }^{12,13}$

\section{STATISTICAL ANALYSIS}

Data were expressed as simple frequencies with percentages or as mean values with standard deviations (mean $\pm \mathrm{SD}$ ). All data were normally distributed, except the FRAX score which was skewed. Categorical data were analyzed using a chi-square test and Fisher's exact test. Numeric variables were analyzed using Student's t-test or the Mann-Whitney U-test (for skewed data). Odds ratios (ORs) for vertebral and hip fracture in relation to TPOAb were determined using unadjusted and adjusted logistic regression (adjusted for age, BMI and BMD, expressed as T-score). In all tests, $p$ value $<0.05$ was considered to be statistically significant. The FRAX score was performed after adjustment for age, BMI and smoking. The ability of the FRAX score and TSH to discriminate between bone fracture positive and negative postmenopausal women was evaluated by the ROC (Receiver Operating Characteristic) curve method. The curves were drawn by plotting the sensitivity against the false positive rate, for varying cut-off of the FRAX score and TSH levels. The area under the curve (AUC) represents a quantitative measure of predictive value of TSH and FRAX score for bone fracture.

\section{RESULTS}

Clinical characteristics of investigated subjects are presented in Table 1 .

Table 1. Characteristics of euthyroid women and women with subclinical hypothyroidism according to TPOAb status

\begin{tabular}{|c|c|c|c|c|c|c|}
\hline \multirow[t]{2}{*}{ Variable } & \multicolumn{2}{|c|}{ Euthyroid } & \multirow[t]{2}{*}{$\mathbf{p}$} & \multicolumn{2}{|c|}{ Subclinical hypothyroid } & \multirow[t]{2}{*}{$\mathbf{p}$} \\
\hline & $\begin{array}{c}\text { TPOAb- } \\
\text { x } \pm \text { sd }\end{array}$ & $\begin{array}{c}\text { TPOAb+ } \\
\mathbf{x} \pm \text { sd }\end{array}$ & & $\begin{array}{c}\text { TPOAb- } \\
\text { x } \pm \text { sd }\end{array}$ & $\begin{array}{c}\text { TPOAb+ } \\
\mathbf{x} \pm \text { sd }\end{array}$ & \\
\hline$n$ & 80 & 30 & & 40 & 39 & \\
\hline Age (yrs) & $60.46 \pm 6.53$ & $61.13 \pm 7.10$ & 0.640 & $59.63 \pm 6.42$ & $58.41 \pm 7.72$ & 0.449 \\
\hline $\mathrm{BMI}\left(\mathrm{m} / \mathrm{kg}^{2}\right)$ & $27.95 \pm 4.26$ & $27.96 \pm 5.72$ & 0.988 & $28.46 \pm 3.73$ & $27.24 \pm 3.05$ & 0.115 \\
\hline Fat mass & $44.27 \pm 5.28$ & $45.56 \pm 6.69$ & 0.605 & $43.04 \pm 6.65$ & $42.52 \pm 7.06$ & 0.807 \\
\hline Menopause onset & $48.90 \pm 4.57$ & $49.03 \pm 3.12$ & 0.883 & $49.33 \pm 3.17$ & $48.10 \pm 3.07$ & 0.898 \\
\hline Smoking & $12(15.0 \%)$ & $2(6.7 \%)$ & 0.243 & $5(12.5 \%)$ & $11(28.2 \%)$ & 0.082 \\
\hline Diabetes mellitus & $3(3.8 \%)$ & $0(0 \%)$ & 0.561 & $3(7.5 \%)$ & $1(2.6 \%)$ & 0.317 \\
\hline Parent fractures & $12(15.0 \%)$ & $1(3.3 \%)$ & 0.091 & $7(17.9 \%)$ & $10(25.6 \%)$ & 0.411 \\
\hline Vitamin D & $60.49 \pm 8.53$ & $60.13 \pm 9.13$ & 0.850 & $60.50 \pm 7.46$ & $59.61 \pm 8.25$ & 0.639 \\
\hline TSH (mU/liter) & $2.28 \pm 0.96$ & $3.46 \pm 1.58$ & $<0.001$ & $7.94 \pm 1.98$ & $8.05 \pm 3.50$ & 0.859 \\
\hline fT4 (mU/liter) & $12.87 \pm 1.59$ & $13.64 \pm 1.35$ & 0.014 & $12.55 \pm 1.51$ & $12.16 \pm 2.60$ & 0.419 \\
\hline LS T-score & $-0.92 \pm 1.57$ & $-1.75 \pm 0.97$ & 0.008 & $-1.23 \pm 1.13$ & $-1.21 \pm 1.04$ & 0.961 \\
\hline Fem neck T- score & $-0.53 \pm 1.08$ & $-1.29 \pm 0.95$ & 0.001 & $-1.06 \pm 0.73$ & $-1.08 \pm 0.67$ & 0.855 \\
\hline FRAX score for major osteop. fr & $4.61 \pm 2.07$ & $6.02 \pm 3.07$ & 0.007 & $6.44 \pm 5.49$ & $6.44 \pm 3.36$ & 0.996 \\
\hline FRAX hip & $0.58 \pm 0.95$ & $1.34 \pm 1.38$ & 0.001 & $1.06 \pm 2.11$ & $1.00 \pm 1.18$ & 0.883 \\
\hline Previous fractures & $2(2.5 \%)$ & $5(16.7 \%)$ & 0.007 & $9(22.5 \%)$ & $9(23.1 \%)$ & 0.951 \\
\hline
\end{tabular}

LS: Lumbar Spine; Fem neck: Femoral neck. 
There were no significant differences in age, BMI and onset of menopause between the groups. Differences in smoking habits and prevalence of diabetes mellitus were not statistically different between the groups. Levels of vitamin D did not differ between the groups.

TSH and fT4 were significantly higher in TPO-

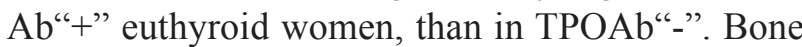
mineral density expressed as $\mathrm{T}$ score was lower at both sites (lumbar spine and femoral neck) in TPOAb" + " euthyroid women. The FRAX score for major osteoporotic fractures and hip FRAX was significantly higher in the euthyroid group with positive TPOAb compared to TPOAb negative, as was the prevalence of previous fractures.

Lower bone mineral density and increased FRAX score for hip and FRAX score for major osteoporotic fractures were associated with presence of TPOAb in euthyroid women in an unadjusted logistic regression model as well as in a model adjusted for age, BMI, vitamin D levels and smoking (Table 2).

In logistic regression analysis, TSH and TPOAb were found to be in significant association with fracture in euthyroid women in an unadjusted model $(\mathrm{p}=0.015 ; \mathrm{OR}=2.012 ; 95 \% \mathrm{CI} 1.147-3.529$ and $\mathrm{p}=0.018 ;$ OR $=7.800 ; 95 \%$ CI 1.424-42.721, respectively) as well as in a model adjusted for age, BMI, vitamin D levels and smoking (Table 3).

TSH was a discriminating predictive factor for fractures in the future (Table 4; Figure 1).

\section{DISCUSSION}

We have shown that euthyroid AITD is associated with decreased bone mineral density at the spine and hip, higher prevalence of fractures and increased risk of future fragility fractures in postmenopausal

Table 4. ROC analysis for TSH and FRAX score in fracture prediction

\begin{tabular}{lcccc}
\hline & Area & SE & $\mathbf{p}$ & $\mathbf{9 5 \%}$ CI for SE \\
\hline TSH & 0.741 & 0.046 & 0.001 & $0.651-0.832$ \\
FRAX main & 0.908 & 0.026 & $<0.001$ & $0.858-0.959$ \\
FRAX hip & 0.741 & 0.058 & 0.001 & $0.628-0.854$ \\
\hline
\end{tabular}

Table 2. Relation of Odds for TPOAb with T and FRAX scores

\begin{tabular}{|c|c|c|c|c|c|c|}
\hline & \multicolumn{3}{|c|}{ Unadjusted logistic regression model } & \multicolumn{3}{|c|}{ Adjusted* logistic regression model } \\
\hline & $\mathbf{p}$ & OR per unit change & 95\% CI for OR & $\mathbf{p}$ & OR per unit change & 95\% CI for OR \\
\hline \multicolumn{7}{|c|}{ T score } \\
\hline LS & 0.010 & 0.612 & $0.421-0.889$ & 0.004 & 0.549 & $0.364-0.828$ \\
\hline Hip & 0.002 & 0.434 & $0.258-0.730$ & 0.001 & 0.350 & $0.189-0.651$ \\
\hline \multicolumn{7}{|c|}{ FRAX score } \\
\hline Total & 0.011 & 1.240 & $1.050-1.465$ & 0.007 & 1.306 & $1.076-1.586$ \\
\hline Hip & 0.008 & 1.759 & $1.160-2.668$ & 0.003 & 2.053 & $1.336-4.325$ \\
\hline
\end{tabular}

* Adjusted for age, BMI, vitamin D levels and smoking

LS=Lumbar Spine

Table 3. Relation of Odds for fracture with thyroid function tests

\begin{tabular}{|c|c|c|c|c|c|c|}
\hline & \multicolumn{3}{|c|}{ Unadjusted logistic regression model } & \multicolumn{3}{|c|}{ Adjusted* logistic regression model } \\
\hline & $\mathbf{p}$ & OR per unit change & $95 \%$ CI for OR & $\mathbf{p}$ & OR per unit change & $95 \% \mathrm{CI}$ for $\mathrm{OR}$ \\
\hline TSH & 0.015 & 2.012 & $1.147-3.529$ & 0.024 & 1.960 & $1.093-3.518$ \\
\hline TPOAb & 0.018 & 7.800 & $1.424-42.721$ & 0.039 & 6.847 & $1.100-42.569$ \\
\hline
\end{tabular}

* Adjusted for age, BMI, vitamin D levels and lumbar spine T-score 


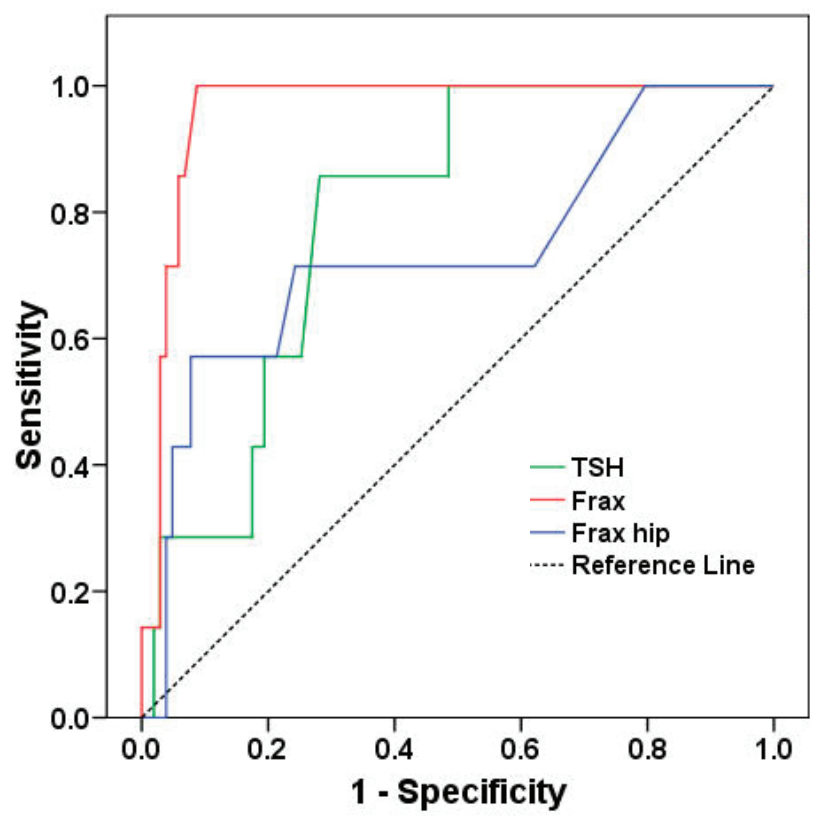

Figure 1. ROC curves for TSH and FRAX score in fracture prediction.

women. Since thyroid autoimmunity per se has been identified as a risk factor for impaired bone quality, it is evident that DXA scans and prevention measures are strongly recommended in these patients.

Given that thyroid hormones are essential for skeleton formation and development, clearly, thyroid dysfunction is a risk factor for low bone mineral density and changes in bone quality. ${ }^{14}$ Hyperthyroidism causes osteopenia and osteoporosis by increasing osteoclast activity and bone turnover, ${ }^{15}$ while hypothyroidism decreases bone remodeling, can provoke falls, slows down secondary bone mineralization and decreases the activity of osteoblasts and osteoclasts. ${ }^{5,6,16-18}$ Even the subclinical form of hypothyroidism and hyperthyroidism are associated with poor bone quality. TSH even moderately below or above the reference range is an independent predictive factor for vertebral fractures. ${ }^{13,15,19-22}$ The HUNT2 study revealed a weak association between mild increase or decrease of TSH and a higher risk of hip fractures, fracture risk being evaluated on the basis of the information given by examiners during the 12.5 years of follow-up. ${ }^{23}$ The same study described an association between TSH in the hyperthyroid range and low bone mineral density in the forearm. ${ }^{24}$ In our previous investigation, in which we used the FRAX score to predict fractures, TSH above the upper reference range was associated with lower femoral neck bone mineral density. ${ }^{13}$

A study in 335 euthyroid Greek postmenopausal women has demonstrated that postmenopausal age and thyroid autoimmunity could predict a vertebral fracture in subjects with normal thyroid function. In this study, and several other investigations, the lower TSH was an independent risk factor for vertebral fractures, as were also antithyroid antibodies, although the levels of T3 and T4 within the reference range had no impact on fracture risk. ${ }^{7,21}$ On the other hand, a cross-sectional study with 37,431 subjects indicated that TSH within the euthyroid or subclinical hypothyroid range correlated with a higher BMD, though $\mathrm{T} 3$ in the upper tertile of the normal range had a negative impact on BMD, but not if $\mathrm{T} 3$ was lower. The authors speculated that the negative effect of T3 on the upper limit has been compensated for by an increase in $\mathrm{TSH}^{25}$

The immune mediators found to play a role in most autoimmune disorders could also be involved in promoting bone loss. Disturbance in osteoclastic activity in new bone formation might be a consequence of chronic inflammatory autoimmune disorders leading to lower bone mineral density, inflammatory cytokines being important regulators of bone destruction. Some of them, such as RANKL, are proosteoclastogenic, others, like OPG, IFN $\gamma$ and IL 4, are antiosteoclastogenic. Mechanisms of bone loss in inflammatory diseases range from influence on systemic control of bone remodeling to direct attack on bone and cartilage by immune cells. ${ }^{8}$ Some investigations have indicated that TSH receptor antibodies (TRAb) are in negative correlation with lumbar spine and femoral neck mineral density. ${ }^{26} \mathrm{~A}$ possible negative impact of TRAb on bone mineral density was reported in the lumbar spine and femoral neck in women with Graves' disease. Bone mineral density was restored after successful stabilization of hyperthyroidism and the investigators concluded that the presence of TRAb could be a good marker of bone impairment during the hyperthyroid phase of Graves' disease. ${ }^{27}$

In our investigation, TPOAb were found to be strongly linked with increased risk of any type of fractures, independently of TSH values. Women in whom TPOAb are present have lower BMD at the 
femoral neck and are at higher fracture risk at any site (Table 2, Table 3). Cut-off value for AITD was $\mathrm{TPOA} b \geq 10 \mathrm{IU} / \mathrm{ml}$. This value was based on the reference range for TSH and TPOAb in the Serbian population, which is in the 95th percentile of the TPOAb upper reference range. ${ }^{28}$ The other investigators used as a discriminant value for TPOAb positive subjects twice the value of the upper reference limit. ${ }^{7,29}$

Autoimmune thyroid disease is often associated with other autoantibodies which are not organ-specific as, for example, antinuclear antibodies (ANA), rheumatoid factor (RF), anticyclic-citrullinated peptides (anti-CCP) and several others. These could form a link between AITD and rheumatoid arthritis and the impact of these two autoimmune diseases on bone quality. ${ }^{30}$ Accumulating evidence suggests that osteoclasts, originating from the monocyte lineage and immune cells share a number of regulatory molecules including cytokines, receptors, signaling molecules and transcription factors, thereby mutually influencing each other. Furthermore, immune cells are derived from bone marrow and thus develop in the same microenvironment as osteoclasts. ${ }^{31,32}$ In fact, patients with excessive activation of the immune system, as in the case of arthritis, are also at higher risk of experiencing concomitant osteoporosis as well as localized bone destruction. A relationship between the immune and skeletal systems has long been recognized. These two systems interact with each other either in physiological or in pathological conditions. Pathological activation of one system affects the other, such as in the case of rheumatoid arthritis where abnormal activation of the immune system affects bone remodeling leading to pathological bone erosions. During chronic inflammation, the balance between bone formation and resorption is skewed towards osteoclast-mediated bone resorption. Moreover, in inflamed joints osteoclasts are located at the interface between the inflamed synovium and the bone and, as in physiological conditions, the major player in bone resorption is the RANKL/RANK/OPG system. ${ }^{33}$ RANKL is produced by several cell types besides osteoblasts, including monocytes, neutrophils, dendritic cells, B and $\mathrm{T}$ lymphocytes. In this way, immune cells have the ability to induce osteoclast differentiation and, consequently, bone resorption. Further, these cells are known for producing a variety of pro-inflammatory cytokines that also contribute to bone damage by potentiating the effects of RANK-RANKL signaling. ${ }^{33}$ The cytokines TNF- $\alpha$ and interleukin (IL)-1, IL- 3, IL-6, IL-7, IL-11, IL-15 and IL-17 potentiate bone loss either by increasing osteoclast generation and activation or by inducing RANKL expression by the osteoblasts. ${ }^{34}$ Cytokines IL- $1 \beta$ and IL-4 are the key players in AITD. The pro-inflammatory effect of IL-1 $\beta$ is based on bone resorption by activation of osteoclasts and encouraged RANKL expression. ${ }^{35,36}$ This study showed a statistically significant positive linear relationship between age, anti-dsDNA, anti-CCP, $\mathrm{RF}$ and duration of thyroiditis with the occurrence of rheumatic diseases, with anti-dsDNA and RF being the most significant predictors. A recent study on hypothyroid/euthyroid postmenopausal women disclosed that TSH positively correlated with OPG and negatively with IL-6 and osteocalcin, moreover that thyroid autoimmunity can affect OPG/RANKL levels independently of hypothyroidism. ${ }^{37}$

Oxydative stress, which is common in several autoimmune diseases as well as in AITD and thyroid dysfunction, could also have an impact on bone mineral density. Loss of balance between antioxidant defence mechanisms and overproduction of free radicals also correlated with thyroid dysfunction. ${ }^{38}$ Considering all the previous data, we can speculate that a proinflammatory microenvironment and oxidative stress in euthyroid postmenopausal women with AITD/positive TPOAb constitutes the mechanism underlying impaired bone quality and increased risk of fracture. Our results additionally indicate that the impact of $\mathrm{TPOAb}$ on bone quality becomes less important when TSH rises above the upper reference range and, furthermore, that elevated TSH increases the risk of any fracture in the future (Table 4, Figure1).

The diagnostic value of bone turnover markers (BTM) in secondary osteoporosis is obsolete with numerous studies having exhibited inconsistent results. ${ }^{39}$ A weak correlation between serum osteocalcin and TSH above the upper reference range in postmenopausal women with previous fractures and increased osteoblastic activity has been described in a previous study of ours in which, incidentally, there was no association between BTM and TPOAb in euthyroid postmenopausal women. ${ }^{13}$ Intestinal resorption of 
vitamin D decreases with aging impairing calcium homeostasis and bone quality. ${ }^{40}$ Both groups of our patients have $25(\mathrm{OH}) \mathrm{D}$ between the insufficiency and the normal range $(50->75 \mathrm{nmol} / \mathrm{L})$.

It should be noted here that our study has a number of limitations. First, there is lack of data regarding duration of autoimmune thyroid disease before inclusion of patients in the study. We also had no information as to whether the fractures occurred before the diagnosis of autoimmune thyroiditis, nor had we collected the data about previous lack of vitamin D and supplementation of vitamin D before inclusion in the study. Finally, information about inflammatory markers with possible influence on bone remodeling was not assessed.

In conclusion, autoimmune thyroid disease is associated with an increased risk of future fractures due to decrease in bone mineral density at the spine and femoral neck in euthyroid postmenopausal women. Presence of TPOAb is a potential marker of higher fracture risk in these patients. However, in subclinical hypothyroidism TSH is a better indicator of future fragility fractures than TPOAb. From the clinical point of view, the significance of this research is reflected in the recommendation to check bone density by DXA in postmenopausal women with AITD in order to identify subjects with increased fragility fractures risk.

\section{REFERENCES}

1. Mazziotti G, Porcelli T, Patelli I, Vescovi PP, Giustina A, 2010 Serum TSH values and risk of vertebral fractures in euthyroid post-menopausal women with low bone mineral density. Bone 46: 747-751.

2. Lakatos P, 2003 Thyroid hormones: Beneficial or deleterious for bone? Calcified Tissue International 73: 205-209.

3. Baddoura R, Hoteit M, El-Hajj Fuleihan G, 2011 Osteoporotic fractures, DXA, and fracture risk assessment: meeting future challenges in the Eastern Mediterranean Region. J Clin Densitom 14: 384-394.

4. Jeremiah MP, Unwin BK, Greenawald MH, Casiano VE, 2015 Diagnosis and management of osteoporosis. Am Fam Physician 92: 261-268.

5. Wojcicka A, Bassett JHD, Williams GR, 2013 Mechanisms of action of thyroid hormones in the skeleton. Biochim Biophys Acta 1830: 3979-3986.

6. Bassett JHD, Williams GR, 2003 The molecular actions of thyroid hormone in bone. Trends Endocrinol Metab
14: 356-364.

7. Lambrinoudaki I, Armeni E, Pliatsika P, et al, 2016 Thyroid function and autoimmunity are associated with the risk of vertebral fractures in postmenopausal women. J Bone Miner Metab. doi:10.1007/s00774016-0752-0

8. Schett G, David JP, 2010 The multiple faces of autoimmune-mediated bone loss. Nat Rev Endocrinol 6: 698-706.

9. Cass AR, Shepherd AJ, Asirot R, Mahajan M, Nizami M, 2016 Comparison of the male osteoporosis risk estimation score (MORES) with FRAX in identifying men at risk for osteoporosis. Ann Fam Med 14: 365369.

10. Ilias I, Spanoudi F, Koukkou E, Nikopoulou SC, 2012 Average of FRAX with BMD and FRAX without BMD use of the FRAX calculator with and without bone mineral density in Greek women. Hormones (Athens) 11: 222-223.

11. Fracture Risk Assessment Tool (FRAX). at $<$ http://www. rheumatology.org/I-Am-A/Rheumatologist/Research/ Clinician-Researchers/Fracture-Risk-Assessment-ToolFRAX $>$

12. Cauley JA, El-Hajj Fuleihan G, Arabi A, et al, 2011 Official positions for FRAX ${ }^{\circledR}$ clinical regarding international differences. J Clin Densitom 14: 240-262.

13. Polovina S, Popovic V, Duntas L, Milic N, Micic D, 2013 Frax score calculations in postmenopausal women with subclinical hypothyroidism. Hormones (Athens) 12: 439-448.

14. Cardoso LF, Maciel LM, de Paula FJ, 2014 The multiple effects of thyroid disorders on bone and mineral metabolism. Arq Bras Endocrinol Metabol 58: 452-463.

15. Cooper DS, Biondi B, 2012 Subclinical thyroid disease. Lancet 379: 1142-1154.

16. Barrett-Connor E, Weiss TW, McHorney CA, Miller PD, Siris ES, 2009 Predictors of falls among postmenopausal women: results from the national osteoporosis risk assessment (NORA). Osteoporos Int 20: 715-722.

17. El Hadidy EHM, Ghonaim M, El Gawad SSA, El Atta MA, 2011 Impact of severity, duration, and etiology of hyperthyroidism on bone turnover markers and bone mineral density in men. BMC Endocr Disord 11: 15.

18. Blum MR, Bauer DC, Collet TH, et al, 2015 Subclinical thyroid dysfunction and fracture risk: a meta-analysis. JAMA 313: 2055-2065.

19. van Rijn LE, Pop VJ, Williams GR, 2014 Low bone mineral density is related to high physiological levels of free thyroxine in peri-menopausal women. Eur J Endocrinol 170: 461-468.

20. Lee WY, Oh KW, Rhee EJ, et al, 2006 Relationship between subclinical thyroid dysfunction and femoral neck bone mineral density in women. Arch Med Res 37: 511-516.

21. Polovina S, Micić D, Miljić D, Milić N, Micić D, Popović V, 2015 The fracture risk assessment tool 
(FRAX $^{\circledR}$ score) in subclinical hyperthyroidism. Vojnosanit Pregl 72: 510-516.

22. Kim BJ, Lee SH, Bae SJ, et al, 2010 The association between serum thyrotropin (TSH) levels and bone mineral density in healthy euthyroid men. Clin Endocrinol (Oxf) 73: 396-403.

23. Svare A, Nilsen TIL, Asvold BO, et al, 2013 Does thyroid function influence fracture risk? Prospective data from the HUNT2 study, Norway. Eur J Endocrinol 169: 845-852.

24. Svare A, Nilsen TIL, Bjoro T, Forsmo S, Schei B, Langhammer A, 2009 Hyperthyroid levels of TSH correlate with low bone mineral density: the HUNT 2 study. Eur J Endocrinol 161: 779-786.

25. Kim THT, Joung JY, Kang M, et al, 2015 A modest protective effect of thyrotropin against bone loss is associated with plasma triiodothyronine levels. PLoS One 10: e0145292.

26. Majima T, Komatsu Y, Doi K, et al, 2006 Negative correlation between bone mineral density and TSH receptor antibodies in male patients with untreated Graves' disease. Osteoporos Int 17: 1103-1110.

27. Amashukeli M, Korinteli M, Zerekidze T, et al, 2013 The negative correlation between thyrotropin receptor-stimulating antibodies and bone mineral density in postmenopausal patients with Graves' disease. J Investig Med 61: 842-847.

28. Žarković M, Ćirić J, Beleslin B, et al, 2011 Further studies on delineating thyroid-stimulating hormone (TSH) reference range. Horm Metab Res 43: 970-976.

29. Shi Y, Sun M, Wang Z, et al, 2014 Association between calcaneus quantitative ultrasound (QUS) parameters and thyroid status in middle-aged and elderly Chinese men with euthyroidism: a population-based cross-sectional study. Endocrine 47: 227-233.

30. Elnady BM, Kamal NM, Shaker RHM, et al, 2016 Prevalence and clinical significance of nonorgan specific antibodies in patients with autoimmune thyroiditis as predictor markers for rheumatic diseases. Medicine (Baltimore) 95: e4336.

31. Takayanagi H, 2007 Osteoimmunology: shared mechanisms and crosstalk between the immune and bone systems. Nat Rev Immunol 7: 292-304.

32. Walsh MC, Kim N, Kadono Y, et al, 2006 OSTEOIMMUNOLOGY: Interplay between the immune system and bone metabolism. Annu Rev Immunol 24: 33-63.

33. Herman S, Krönke G, Schett G, 2008 Molecular mechanisms of inflammatory bone damage: emerging targets for therapy. Trends Mol Med 14: 245-253.

34. Fonseca JE, Cortez-Dias N, Francisco A, et al, 2016 Inflammatory cell infiltrate and RANKL/OPG expression in rheumatoid synovium: comparison with other inflammatory arthropathies and correlation with outcome. Clin Exp Rheumatol 23: 185-192.

35. Zaaber I, Mestiri S, Hammedi H, et al 2016 Association of interleukin- $1 \mathrm{~b}$ and interleukin-4 gene variants with autoimmune thyroid diseases in Tunisian population. Immunol Invest 45: 284-297.

36. Feng X, McDonald JM, 2011 Disorders of bone remodeling. Annu Rev Pathol Mech Dis 6: 121-145.

37. Konca Degertekin C, Turhan Iyidir O, Aktas Y1lmaz B, et al, 2016 RANKL/osteoprotegerin system and bone turnover in hashimoto thyroiditis. Calcif Tissue Int 99: 365-372.

38. Ruggeri RM, Vicchio TM, Cristani M, et al 2016 Oxidative stress and advanced glycation end products in Hashimoto's thyroiditis. Thyroid 26: 504-511.

39. Biver E, Chopin F, Coiffier G, et al, 2012 Bone turnover markers for osteoporotic status assessment? A systematic review of their diagnosis value at baseline in osteoporosis. Joint Bone Spine 79: 20-25.

40. Veldurthy V, Wei R, Oz L, Dhawan P, Jeon YH, Christakos S, 2016 Vitamin D, calcium homeostasis and aging. Bone Res 4: 16041. 